Programa de Pós-Graduação em Engenharia de Produção - PPGEP

Laboratório de Qualidade de Vida - LaQVida

Universidade Tecnológica Federal do Paraná - UTFPR

Ponta Grossa - PR - Brasil

v. 03 , n. 02 , jul./dez. 2011, p. 13-22

DOI: $10.3895 / \mathrm{S} 2175-08582011000200002$

\section{REVISTA BRASILEIRA DE QUALIDADE DE VIDA}

\title{
Qualidade de vida do estudante do ensino médio e tabagismo
}

\section{Quality of life of high school students and smoking}

\author{
Isaac Rosa Marques \\ Universidade de Santo Amaro - UNISA - São Paulo - Brasil \\ imarques@unisa.br \\ Fabiana Arandas \\ Universidade de Santo Amaro - UNISA - São Paulo - Brasil \\ fabi.arandas@bol.com.br
}

\begin{abstract}
Resumo
O objetivo do estudo foi identificar o nível de Qualidade de Vida (QV) de estudantes do ensino médio de uma escola estadual localizada na Região Sul da cidade de São Paulo e associar estes resultados com o hábito de fumar. Tratou-se de uma pesquisa de corte transversal realizada com com 144 estudantes do nível médio. Foi utilizado o questionário WHOQoL Abreviado para a coleta de dados. Para análise dos resultados estabeleceu-se os seguintes parâmetros: QV ruim= de 0 a 1,500; QV intermediária= de 1,501 a 2,500; QV boa= de 2,501 a 3,000 pontos. Os resultados demonstraram que os estudantes têm QV intermediária. O domínio Físico foi o de pior desempenho no questionário (QV ruim - média=1,451), enquanto que o domínio de melhor desempenho foi o Psicológico $(\mathrm{QV}$ intermediária - média=2,409). O grupo de fumantes teve o pior desempenho no domínio Físico (média=0,997). Concluiu-se que o tabagismo tem associação com o domínio Físico e influencia o nível de QV.
\end{abstract}

Palavras-chave: qualidade de vida, estudantes, tabagismo.

\begin{abstract}
The purpose of this study was to identify the level of quality of life (QoL) of high school students from a state school located in the Southern Region of the city of São Paulo and to associate those results with smoking habit. That was a cross-sectional research carried out with 144 high school students. The WHOQOL-BREF questionnaire was used for data collection. To analyze the results it was established the following parameters: poor $\mathrm{QoL}=0$ to 1.500 ; intermediate $\mathrm{QoL}=1.501$ to 2.500; good QoL=2.501 to 3.000 points. The results showed that students have an intermediate QoL. The physical domain being the worst performance in the questionnaire (poor QoL - average=1.451), while in the Psychology domain the best performance was observed (intermediate QoL - average $=2.409$ ). The smoking group had the worst performance in the Physical domain (average=0.997). It was concluded that smoking has association with Physical domain and influences the level of QoL.

Keywords: quality of life, students, smoking.
\end{abstract}

\section{Introdução}

A qualidade de vida (QV) tem sido objeto de diversos estudos nos últimos anos (MINAYO; 
HARTZ; BUSS, 2000). As investigações sobre a QV abrangem diversos segmentos da vida da sociedade, desde as situações de doença às situações do cotidiano, como por exemplo, as repercussões do trabalho, da exposição a agentes prejudiciais, aos traumas decorrentes de diversas situações. Estudar a QV permite obter dados precisos para que novas alternativas possam ser implementadas no sentido de melhorar esta QV. Todas estas investigações têm sido fundamentais para que melhorias possam ser implementadas em todos os âmbitos da vida humana (DANTAS; SAWADA; MALENKO, 2003).

Um dos focos principais dos estudos sobre a QV é, inegavelmente, os problemas causados pelas doenças crônicas (MARTINS; FRANÇAS; KIMURA, 1996). Entretanto, investigações sobre as condições de vida consideradas normais vêm acontecendo nos últimos anos. Diversos estudos apresentaram as relações que o sistema de vida pode desempenhar na QV (VECCHIA; RUIZ; BOCCHI, 2005).

Entre as populações estudadas, está a população de estudantes dos diversos níveis. Vários estudos enfocam a vida estudantil universitária (SOUZA; MARQUES, 2010) e poucos estudos a QV do estudante nível médio.

O tabaco e o tabagismo são responsáveis por diversas doenças, algumas delas de aparecimento em longo prazo (ARAÚJO; MENEZES; DOREA, 2004). Estima-se que na atualidade há no mundo 1,3 bilhão de fumantes, dos quais $80 \%$ vivem em países em desenvolvimento.

No Brasil, esses números estão em torno de 25 milhões (CÂMARA JÚNIOR, 2005). Cerca de seis em cada dez crianças já fumaram. Somando todos os jovens em idade escolar, ou seja, entre 10 e 18 anos, cerca de três milhões já estão completamente dependentes da nicotina.

A prevalência e os fatores de riscos para o tabagismo em adolescentes de 10 a 19 anos em fase escolar, caracterizada pela aventura proporcionada por novas experiências, mas que culmina com o vício no avançar da idade. Para muitos fumantes, esta motivação ocorrida na adolescência, foi o início de uma vida no vício (MALCON; MENEZES; CHATKIN, 2003).

Ao deparar-se com o tema da QV relacionado ao tabagismo, que seria um dos problemas de saúde pública, o profissional enfermeiro poderá orientar tratamentos ou formas para o abandono do tabagismo (BRASIL, 2002). Este profissional tem sua atuação como agente de educação nos vários âmbitos da atenção básica. No contexto da escola, por exemplo, pode o profissional atuar como educador para a promoção de saúde. Portanto, o conhecimento de possíveis causas que possam interferir na QV, pode ser útil para desenvolver programas de saúde voltados para os sujeitos que frequentam a escola. Conhecer também as influências que o tabagismo acarreta na QV pode contribuir para o corpo de conhecimentos para melhorar as estratégias de combate ao tabagismo entre esta população. As entidades médicas e órgãos de saúde devem garantir a aplicação das leis antitabaco e a enfermagem realizando campanhas preventivas enfocando os malefícios do tabagismo na adolescência assim como a monitorização periódica do efeito de tais campanhas através de pesquisas de base populacional (MALCON; MENEZES; MAIA, 2003).

Neste sentido, este estudo teve como objetivo identificar a QV de estudantes do ensino médio de uma escola estadual localizada na Região Sul da cidade de São Paulo e associar estes resultados com hábito de fumar.

\section{Método}

Tratou-se de uma pesquisa de corte transversal constituída com a participação de 144 estudantes do nível médio de uma escola estadual localizada na Região Sul da cidade de São Paulo.

Foram incluídos os alunos sem restrição de idade e que estavam matriculados no Ensino Médio no ano letivo de 2011, de ambos os sexos. A amostragem foi constituída pela técnica não probabilística de conveniência.

Foi utilizado o instrumento WHOQoL Abreviado da World Health Organization Quality of Life Group por ser considerado um instrumento validado e que abrange o conteúdo o qual é objeto de estudo desta pesquisa. A ausência de um instrumento que avaliasse QV per se, com uma 
perspectiva internacional, fez com que a Organização Mundial de Saúde construísse um Grupo de QV com a finalidade de desenvolver instrumentos capazes de fazê-lo dentro de uma perspectiva transcultural (THE WHOQOL GROUP, 1995).

$\mathrm{O}$ instrumento WHOQoL Abreviado contém 26 questões que abrangem os seguintes domínios: auto-avaliação da QV em si; abordagem sobre a saúde; locomoção/transporte, atividades do dia-a-dia, relacionamento pessoal, sexualidade, condições de moradia, acesso aos serviços de saúde, aspectos psicológicos e expectativa em relação ao futuro.

A coleta de dados foi realizada no ambiente da referida escola durante o período matutino e noturno. Previamente à coleta de dados, os participantes foram informados dos objetivos da pesquisa assim como os critérios de sua participação. Os alunos maiores de 18 anos e que aceitaram participar assinaram o Termo de Consentimento Livre e Esclarecido (TCLE). Os alunos com menos de 18 anos levaram a Carta de Informação e o TCLE para os pais assinarem. Em outra oportunidade previamente agendada, foi feita a coleta de dados.

O projeto de pesquisa foi apreciado pela direção da referida escola obtendo aprovação e em seguida foi aprovado pelo Comitê de Ética em Pesquisa da Universidade de Santo Amaro, sob o parecer $\mathrm{n}^{\mathrm{o}}$ 168/2010 (CAAE 5635.0.000.386-10).

Para a análise dos dados referentes aos domínios do WHOQoL Abreviado foi desenvolvida uma planilha no aplicativo MS Excel® para o cálculo dos escores. O balizamento dos resultados estabelecido foi: de 0 a 1,50=QV ruim; de 1,51 a 2,50=QV intermediária; de 2,51 a 3,00=QV boa, conforme Quadro 1:

Quadro 1 - Interpretação dos resultados dos domínios da QV
\begin{tabular}{|l|l|}
\hline \multicolumn{1}{|c|}{ Escores médios } & \multicolumn{1}{c|}{ Nível de Qualidade de Vida } \\
\hline 0 a 1,500 & QV ruim \\
\hline 1,501 a 2,500 & QV intermediária \\
\hline 2,501 a 3,000 & QV boa \\
\hline
\end{tabular}

Fonte: Adaptado de Fleck et al. (2000).

$\mathrm{Na}$ planilha os dados foram agrupados pelas questões referentes aos domínios de acordo com o grupo de fumantes e não fumantes. O domínio Físico abrangeu as questões três a cinco; o domínio Psicológico as questões seis a dez; o domínio Nível de independência as questões 11 a 14; o domínio Relações sociais as questões 15 a 17; o domínio Ambiente as questões 18 a 25 e o domínio Aspectos Espirituais a questão 26.

Completando a análise, foram calculados os valores globais e dos grupos para média, variância e o desvio padrão (DP) de cada domínio.

\section{Resultados e Discussão}

\subsection{Perfil da Amostra Participante}

A pesquisa foi realizada nos meses de fevereiro a março de 2011, com estudantes de uma escola estadual da Região Sul da cidade de São Paulo. Esta escola funciona no período matutino e noturno. A escola tem atualmente 634 alunos entre o Ensino Fundamental e Ensino Médio. Deste total, 2/3 são estudantes do Ensino Médio, os quais eram sujeitos de interesse para a pesquisa.

Participou da pesquisa uma amostra de 144 estudantes, sendo 40 do período matutino e 104 do período noturno. A maioria da amostra $(72,2 \%)$ era do período noturno devido ao fato de que houve muita dificuldade de obter a autorização dos pais ou responsáveis ou os estudantes esqueceram-se de trazer o TCLE assinado pelos pais.

Quanto à faixa etária, 36,1\% pertencia a faixa dos 13 aos 20 anos, 25,7\% à faixa etária dos 21 aos 30 anos e $38.2 \%$ à faixa etária maior que 30 anos (Tabela 1). Este resultado evidencia que há um grande contingente de alunos cursando o Ensino Médio com idade maior que 30 anos, o que significa um atraso considerável nos estudos. Este perfil de faixa etária ainda é comumente visto nas 
escolas de Ensino Médio, sobretudo nas públicas (CALAIS; ANDRADE; LIPP, 2003).

Quanto ao sexo, a maioria dos estudantes pertence ao sexo feminino, mesmo considerando o período de estudos. Era de se esperar um menor número de estudantes do sexo feminino estudando no período noturno, mas nos dias atuais observa-se a presença de estudantes do sexo feminino em mesmo número que de estudantes do sexo masculino, independentemente do período de estudos (CALAIS; ANDRADE; LIPP, 2003).

Da amostra participante, $56,9 \%$ dos estudantes trabalham paralelamente aos estudos. Isto se acentua nos estudantes do período noturno. Este dado exerce certa influência na QV geral do estudante, conforme pesquisa de Souza e Marques (2010), na qual ficou evidenciado que a associação trabalho e estudos tende a prejudicar a QV como um todo.

Tabela 1 - Variáveis demográficas dos participantes do estudo

\begin{tabular}{lcc}
\hline Faixa etária (em anos) & $\mathbf{N}$ & \% \\
$\quad$ 13 a 20 & 52 & 36,1 \\
21 a 30 & 37 & 25,7 \\
$\quad$ > 30 & 55 & 38,2 \\
Sexo & & \\
$\quad$ Masculino & 47 & 32,6 \\
$\quad$ Feminino & 97 & 67,4 \\
Período dos estudos & & \\
$\quad$ Matutino & 40 & 27,8 \\
$\quad$ Noturno & 104 & 72,2 \\
Trabalho paralelo aos estudos & & \\
$\quad$ Sim & 82 & 56,9 \\
$\quad$ Não & 62 & 43,0 \\
Tabagismo & & 11,1 \\
$\quad$ Sim & 16 & 88,9 \\
$\quad$ Não & 128 & \\
\hline
\end{tabular}

Fonte: Autoria própria (2011).

Quanto ao tabagismo, verificou-se que $11,1 \%$ dos estudantes referiu ter o hábito de fumar. Isto evidencia a influência das campanhas antitabagismo amplamente divulgas na mídia e que atinge as escolas de um modo geral. Em um estudo realizado com escolares do Ensino Fundamental, a taxa de fumantes encontrada foi de 3,2\% (BORDIN et al., 1992). Portanto, menor que neste estudo. Mas, se considerado o Nível de independência, a taxa de fumantes deste estudo não pode ser considerada alta. Por outro lado, o estudo de Cordeiro et al. (2010), apresentou um resultado da prevalência do tabagismo muito semelhante ao deste estudo $(12,9 \%)$.

Ainda abordando o Nível de independência, o que favorece o uso do cigarro é o fato de que os alunos que são trabalhadores têm condição financeira para comprar o cigarro, enquanto que estudantes que não têm remuneração não o fazem com tanta facilidade (SOUZA; SILVEIRA FILHO, 2007).

\subsection{Análise da Qualidade de Vida do Estudante segundo os Domínios}

A QV dos estudantes foi avaliada a partir da aplicação do questionário WHOQoL Abreviado. A análise foi feita segundo os domínios: Físico, Nível de independência, Relações sociais, Ambiente e Aspectos espirituais. Como variável de comparação, foi selecionado o fator tabagismo, decorrendo a formação de dois grupos: não fumantes e fumantes.

Os dados do questionário foram tabulados em uma planilha do MS Excel, organizando os domínios segundo as questões. Em seguida, foram produzidas as médias por questão e, em seguida, somando-se as médias das questões referentes a cada domínio. Dessa forma foram produzidas as médias de cada domínio. Os resultados de cada grupo foram analisados em planilhas separadas. As médias dos domínios estão apresentadas na Tabela 2. A média global estabelecida foi de 2,060, a média do grupo de não fumantes foi de 2,130 e do grupo de fumantes foi de 1,990. Assim também 
foi calculada a variância e o DP, que resultou no valor de 0,065 . Na Tabela 3, estão apresentadas a variância e o DP por domínio e por grupo.

\subsubsection{Domínio Físico}

O resultado da média global do domínio Físico foi $1,451(\mathrm{DP}=-0,674)$ indicando uma QV ruim para todos os participantes da pesquisa. $O$ resultado médio $1,906(\mathrm{DP}=-0,154)$ verificado no grupo de não fumantes indicou QV intermediária, enquanto que o grupo de fumantes apresentou um resultado de $0,997(\mathrm{DP}=-1,128)$, indicando $\mathrm{QV}$ ruim.

Tabelas 2 - Médias dos domínios do WHOQoL Abreviado segundo grupo de estudantes

\begin{tabular}{lccc}
\hline \multirow{2}{*}{ Domínios } & \multicolumn{3}{c}{ Médias } \\
\cline { 2 - 4 } & Não fumantes $(\mathbf{n = 1 2 8})$ & Fumantes $(\mathbf{n = 1 6})$ & Global $(\mathbf{n}=\mathbf{1 4 4})$ \\
\hline Físico & 1,906 & 0,997 & 1,451 \\
Psicológico & 2,409 & 2,487 & 2,448 \\
Nível de independência & 2,171 & 2,062 & 2,116 \\
Relações sociais & 2,091 & 2,000 & 2,045 \\
Ambiente & 2,305 & 2,328 & 2,316 \\
Aspectos Espirituais & 1,898 & 2,125 & 2,011 \\
\hline
\end{tabular}

Fonte: Autoria própria (2011).

Tabela 3 - Variância e DP observados nos grupos de não fumantes e fumantes

\begin{tabular}{lcccc}
\hline \multirow{2}{*}{ Domínios } & \multicolumn{2}{c}{ Não fumantes $(\mathbf{n = 1 2 8})$} & \multicolumn{2}{c}{ Fumantes $(\mathbf{n}=\mathbf{1 6})$} \\
\cline { 2 - 5 } & Variância & DP & Variância & DP \\
\hline Físico & $-0,154$ & $-0,219$ & $-1,063$ & $-1,128$ \\
Psicológico & 0,349 & 0,283 & 0,427 & 0,361 \\
Nível de independência & 0,111 & 0,045 & 0,002 & $-0,063$ \\
Relações sociais & 0,031 & $-0,034$ & $-0,060$ & $-0,125$ \\
Ambiente & 0,245 & 0,179 & 0,268 & 0,202 \\
Aspectos Espirituais & $-0,162$ & $-0,227$ & 0,065 & 0,000 \\
\hline
\end{tabular}

Fonte: Autoria própria (2011).

Vários aspectos podem ter influência negativa para o resultado da média global, o mais comum é a falta de realização de atividades físicas regulares. Vários estudos sobre QV atribuem mau desempenho neste domínio devido a não realização de atividades físicas regulares. Além disto, considerando os sujeitos de estudo, sabe-se que a vida nesta fase é permeada de agitação, como festas de final de semana, sono alterado, má alimentação, entre outros.

No estudo de Silva e Malina (2000) foi verificado o nível de atividade física entre estudantes do nível médio, observando um alto grau de sedentarismo. Evidentemente, o nível de sedentarismo é maior entre estudantes do sexo feminino. Os resultados observados nesta pesquisa evidenciaram que o tabagismo exerceu uma influência complementar no prejuízo à QV neste domínio.

\subsubsection{Domínio Psicológico}

O resultado da média global foi de 2,448 ( $\mathrm{DP}=0,322)$ para o domínio Psicológico, indicando uma QV intermediária para todos os participantes da pesquisa. $\mathrm{O}$ resultado médio do grupo de não fumantes foi de 2,409 $(0,283)$ e do grupo de fumantes 2,487 ( $\mathrm{DP}=0,361)$, ambos os grupos têm $\mathrm{QV}$ intermediária.

$\mathrm{Na}$ avaliação deste domínio, vários fatores podem influenciar a QV. Não foi percebida influência intrínseca do tabagismo na QV do grupo de fumantes. Na verdade, os aspectos psicológicos são permeados por diversas peculiaridades, desde as influências familiares, nível socioeconômico, perfil individual, relações pessoais, entre outros.

No estudo de Rocha et al. (2006), foi verificado que os sintomas psicológicos mais comuns 
incluem aqueles indicativos de estado depressivo. $\mathrm{O}$ aparecimento destes sintomas é progressivo à medida que ocorre progressão escolar que encaminha para a escolha de uma profissão ou curso superior, o que é exacerbado também pelo exame de admissão nas universidades (vestibular). Os sintomas depressivos também são mais frequentes em estudantes do sexo feminino. No presente estudo, a correlação destas variáveis não foi estudada.

\subsubsection{Domínio Nível de Independência}

O domínio Nível de independência diz respeito à autonomia que o indivíduo tem na tomada de decisão das questões de sua própria vida. $O$ resultado da média global foi de 2,116 $(\mathrm{DP}=-0,009)$, indicando uma QV intermediária. $\mathrm{O}$ grupo de não fumantes teve o resultado da média de $2,171(\mathrm{DP}=0,045)$ e o grupo de fumantes o resultado de $2,062(\mathrm{DP}=-0,063)$, indicando o mesmo nível de QV.

Uma característica que pode sustentar este resultado foi o dado de maior frequência na variável faixa etária. $\mathrm{Na}$ amostra de pesquisa, 38,2\% tinha idade > 30 anos e 25,7\% idade entre 21 a 30 anos, o que sugere que estes estudantes já têm uma autonomia em relação às decisões da sua própria vida. A representação desta amostra $(n=107)$ no grupo pode ter forçado o resultado para que tivessem a avaliação da QV no nível intermediário. Nesse sentido, Togni e Carvalho (2007) verificaram que a opção por estudar à noite é uma tendência no Ensino Médio no Brasil, o que pode forçar o estudante a ter mais autonomia em relação à sua vida.

\subsubsection{Relações Sociais}

As relações pessoais desempenham um importante papel na vida do ser humano e também é uma influência determinante na QV. O resultado da média global neste domínio foi de 2,045 (DP= 0,080), indicando QV intermediária. O grupo de não fumantes teve média de 2,091 $(\mathrm{DP}=-0,034)$, enquanto o grupo de fumantes média de 2,000 ( $\mathrm{DP}=-0,125)$.

Considerando que o nível de QV verificado na amostra, pode-se associar com as seguintes variáveis: trabalho paralelo aos estudos, verificado em $56,9 \%$ e o período de estudos noturno $72,2 \%$. O trabalho paralelo aos estudos indica que o estudante fica pouco tempo em casa, o que reduz o seu relacionamento social familiar. Evidentemente, a qualidade de um relacionamento fora do âmbito familiar é questionável, pois pode ocorrer somente nas relações de trabalho ou ainda no ambiente escolar. Por isso, pode ter influência negativa no resultado da média global.

Com relação ao tabagismo, no estudo de Prado e Silva et al. (2008), os autores pesquisaram sobre fatores que podem influenciar na iniciação do tabagismo e um deles é a deterioração das relações familiares. As relações dentro da família têm suas implicações na vida escolar também são apontadas por Carvalho (2000). No caso de um relacionamento considerado repressivo, o mesmo pode ocasionar no estudante a motivação para a realização de atividades não permitidas no ambiente doméstico, um deles é a experimentação do fumo (PINTO; RIBEIRO, 2007; PASQUALOTTI et al., 2006; ZANINI et al., 2006).

\subsubsection{Ambiente}

O domínio Ambiente produziu o resultado da média global de 2,316 (DP=0,190), indicando QV intermediária. $\mathrm{O}$ grupo de não fumantes teve a média de 2,305 ( $\mathrm{DP}=0,179)$ enquanto que o grupo de fumantes a média de 2,328 ( $\mathrm{DP}=0,202)$. Não houve neste domínio uma diferença importante entre os resultados, o que indica que não é possível estabelecer uma relação direta com o nível de QV entre os participantes da pesquisa. A realidade da maioria da amostra está associada aos locais que frequentam, no caso a escola, o local de trabalho ou ainda o ambiente domiciliar. resultados.

Não foram encontrados estudos na literatura nacional sobre esta abordagem para discutir tais 


\subsubsection{Aspectos Espirituais}

Os aspectos espirituais também desempenham um importante papel na QV. Neste domínio, o resultado da média global foi o segundo mais baixo: 2,011 $(\mathrm{DP}=-0,114)$, ficando atrás somente do domínio Físico. O grupo de não fumantes teve o pior desempenho entre todos os domínios (média $=1,898 / \mathrm{DP}=-0,162)$ e o grupo de fumantes média 2,125 (DP=0). Como explicar esse resultado?

Os aspectos espirituais abrangem a vida de um modo muito amplo e repercutem na forma com se encara a vida. Ter ou não ter uma religiosidade influencia a QV neste domínio. Considerando a faixa etária da amostra que participou deste estudo, pode-se inferir que a religiosidade não é um tópico importante na vida desses estudantes. Obviamente, é sabido que à religiosidade ou espiritualidade tem sido atribuído, mais recentemente, um valor no que se refere à QV (PANZINI et al., 2007; PANZINI et al., 2011).

É importante ressaltar que houve uma diferença considerável nos valores médios neste domínio. Conforme mostra a Figura 1, observa-se que o domínio Físico (F) foi o que apresentou resultados mais discrepantes em relação ao DP; ficando em segundo lugar o domínio Aspectos espirituais (AE). Isoladamente, este foi o domínio de pior desempenho do grupo de não fumantes, não sendo possível estabelecer uma associação com o tabagismo em si.

Figura 1 - Evolução dos desvios padrão por domínios entre os grupos de não fumantes e fumantes

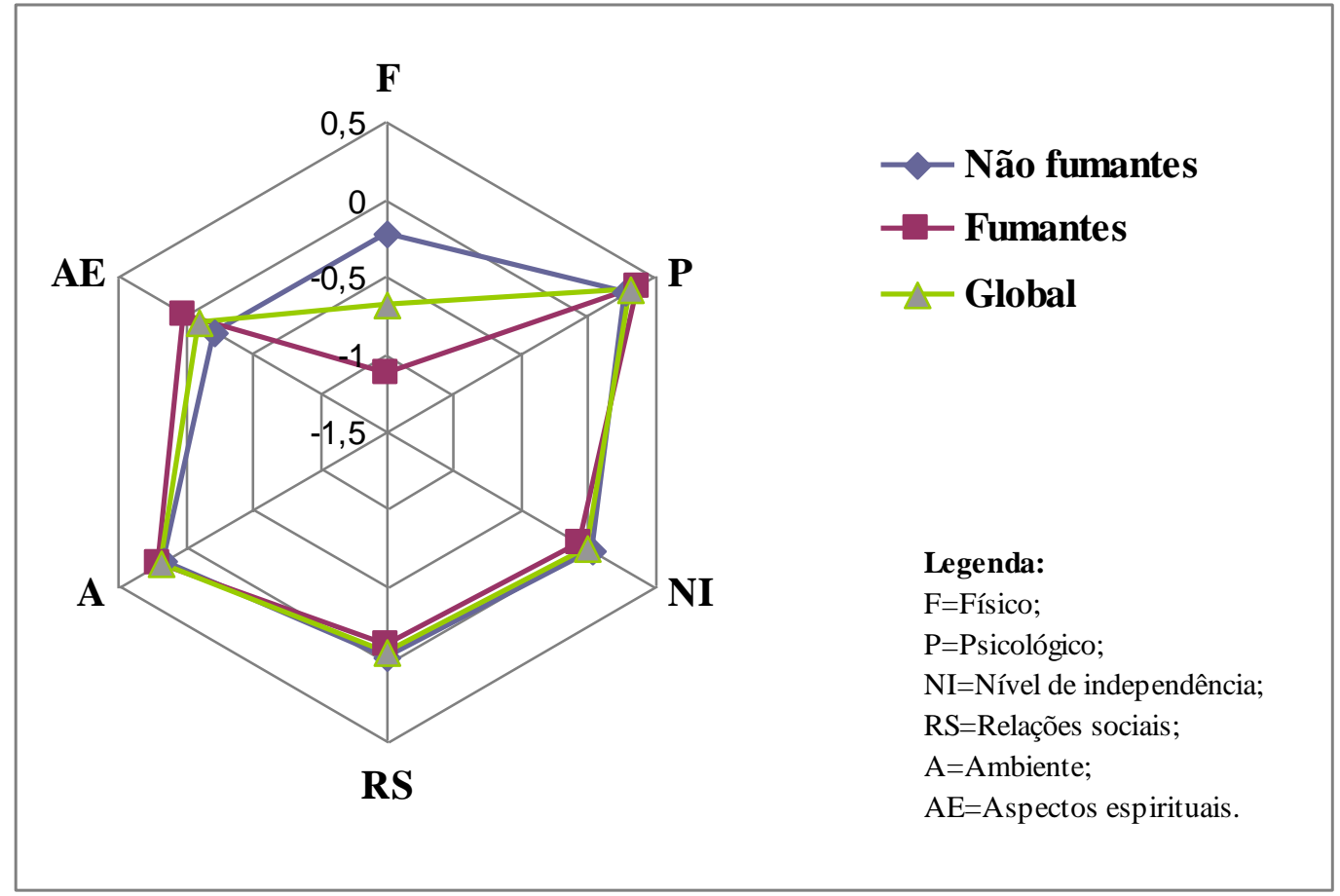

Fonte: Autoria própria (2011).

\section{Conclusão}

Esta pesquisa objetivou identificar a QV de estudantes do ensino médio e associar os resultados com o hábito de fumar. Mediante os resultados apresentados, conclui-se que a QV dos estudantes que participaram da pesquisa situa-se no nível intermediário.

O domínio Físico foi o que apresentou a pior qualidade (QV ruim, média 1,451/DP= 0,674). Este resultado tem associação com o tabagismo, embora ambos os grupos tivessem desempenho igualmente abaixo da média ou do DP (não fumantes: média 1,906/DP= -0,219; fumantes: média=0,997/DP=-1,128), este foi o pior desempenho em todos os domínios para o 
grupo de fumantes.

No domínio Psicológico, foi verificada a maior média $(2,448)$, mas ainda no nível de QV intermediária. Por outro lado, o grupo de não fumantes apresentou o pior desempenho no domínio Aspectos espirituais (média=1,898), sendo a pior média de todos os domínios.

Em relação ao objetivo específico que era estabelecer associação entre QV e o tabagismo, foi evidenciado que o tabagismo tem associação direta com a QV dos estudantes do nível médio que participaram da pesquisa.

\section{Considerações Finais e Recomendações}

O contexto do Ensino Médio abre espaço para a atuação de enfermagem na atenção básica à saúde. Muito embora o governo venha exercendo ampla influência nas campanhas contra o tabagismo, existe a necessidade de intervenção dos enfermeiros que atuam na área de atenção básica à saúde no sentido de promoverem campanhas antifumo nas escolas que pertencem à sua área de atuação.

Estes programas podem contribuir para que os jovens estudantes do ensino médio melhorem suas condições de vida, tendo maior QV. Esta contribuição poderá influenciar vários dos domínios da QV, não somente os aspectos físicos.

A recomendação principal que pode ser deixada com este estudo é que os enfermeiros que atuam na área de atenção básica à saúde, quer seja nas Unidades Básicas de Saúde ou na Estratégia de Saúde da Família, que planejem ações com programas antitabagismo e também com informações sobre a saúde para esta população em específico. Estas ações voltadas para os jovens que estejam no ensino médio deverão enfocar a necessidade de se buscar um nível melhor de QV.

\section{Referências}

ARAÚJO, A. J.; MENEZES, A. M. B.; DOREA, A. P. S. Diretrizes para Cessação do Tabagismo. Jornal Brasileiro de Pneumologia, São Paulo, v. 30, sup. 2, p. S1-S7, ago. 2004.

BORDIN, R. et al. Prevalência de tabagismo entre escolares em município de área metropolitana da Região Sul, Brasil, 1991. Cadernos de Saúde Pública, Rio de Janeiro, v. 9, n. 2, p. 185-189, abr./jun. 1992.

BRASIL. MINISTÉRIO DA SAÚDE. Portaria GM/MS/Nº1575, de 29 de agosto de 2002. Consolida o Programa Nacional de Controle de Tabagismo, e dá outras providências. Diário Oficial da União, Brasília, seção 1, p. 42-47, set. 2, 2002.

CALAIS, S. L.; ANDRADE, L. M. B.; LIPP, M. E. N. Diferenças de sexo e escolaridade na manifestação de stress em adultos jovens. Psicologia, Reflexão e Crítica, Porto Alegre, v. 16, n. 2, p. 257-263, mai./ago. 2003.

CÂMARA JÚNIOR, J. P. O tabagismo como um problema de saúde pública. Revista Brasileira de Promoção da Saúde, Fortaleza, v. 18, n. 3, p. 115-116, set. 2005.

CARVALHO, M. E. P. Relações entre família e escola e suas implicações de gênero. Cadernos de Pesquisa, São Paulo, n. 110, p. 143-155, jul. 2000.

CORDEIRO, E. A. K.; KUPEK, E.; MARTINI, J. G. Prevalência do tabagismo entre escolares de Florianópolis, SC, Brasil e as contribuições da enfermagem. Revista Brasileira de Enfermagem, Brasília, v. 63, n. 5, p. 706-711, set./out. 2010. 
DANTAS, R. A. S.; SAWADA, N. O.; MALENKO, M. B. Pesquisa sobre qualidade de vida: revisão da produção científica das universidades públicas do Estado de São Paulo. Revista LatinoAmericana de Enfermagem, Ribeirão Preto, v. 11, n. 4, p. 532-538, jul./ago. 2003.

FLECK, M. P. A. et al. Aplicação da versão em português do instrumento abreviado de avaliação da qualidade de vida "WHOQOL-bref”. Revista de Saúde Pública, São Paulo, v. 34. n. 2, p. 178-183, abril. 2000.

MALCON, M. C.; MENEZES, A. M. B.; CHATKIN, M. Prevalência e fatores de risco para tabagismo em adolescentes. Revista de Saúde Pública, São Paulo, v. 37, n. 1, p. 1-7, jan/fev. 2003.

MALCON, M. C.; MENEZES, A. M. B.; MAIA, M. F. Prevalência e fatores de risco para tabagismo em adolescentes na América do Sul: uma revisão sistemática da literatura. Revista Panamericana de Salud Pública, Washington, v. 13, n. 4, p. 222-228, abr. 2003.

MARTINS, L. M.; FRANÇAS, A. P. D.; KIMURA, M. Qualidade de vida de pessoas com doença crônica. Revista Latino-Americana de Enfermagem, Ribeirão Preto, v. 4, n. 3, p. 5-18, dez. 1996.

MINAYO, M. C. S.; HARTZ, Z. M. A.; BUSS, P. M. Qualidade de vida e saúde: um debate necessário. Ciência e Saúde Coletiva, Rio de Janeiro, v. 5, n. 1, p. 7-18, jan./jun. 2000.

PANZINI, R. G. et al. Validação do Instrumento de Qualidade de Vida/espiritualidade, religião e crenças pessoais. Revista de Saúde Pública, São Paulo, v. 45, n. 1, p. 153-165, fev. 2011.

PANZINI, R. G. et al. Qualidade de vida e espiritualidade. Revista de Psiquiatria Clínica, São Paulo, v. 34, sup. 1, p. 105-15, 2007.

PASQUALOTTI, A. et al. Experimentação de fumo em estudantes do ensino fundamental e médio de área urbana na Região Sul do Brasil. Revista Interamericana de Psicología, La Rioja, v. 40, n. 2, p. 215-218, may./ago. 2006.

PINTO, D. S.; RIBEIRO, A. S. Variáveis relacionadas à iniciação do tabagismo entre estudantes do ensino médio de escola pública e particular na cidade de Belém - PA. Jornal Brasileiro de Pneumologia, São Paulo, v. 33, n. 5, p. 558-564, nov./dez. 2007.

PRADO E SILVA, M.; SILVA, R. M. V. G.; BOTELHO, C. Fatores associados à experimentação do cigarro em adolescentes. Jornal Brasileiro de Pneumologia, São Paulo, v. 34, n. 1, jan./fev. 2008.

ROCHA, T. H. R. et al. Sintomas depressivos em adolescentes de um colégio provado. Psicologia USF, Itatiba, v. 11, n. 1, p. 95-102, jan./jun. 2006.

SILVA, R. C. R.; MALINA, R. M.. Nível de atividade física em adolescentes do Município de Niterói, Rio de Janeiro, Brasil. Cadernos de Saúde Pública, Rio de Janeiro, v. 16, n. 4, p. 10911097, out./dez. 2000.

SOUZA, D. P. O.; SILVEIRA FILHO, D. X. Uso recente de álcool, tabaco e outras drogas entre estudantes adolescentes trabalhadores e não trabalhadores. Revista Brasileira de Epidemiologia, São Paulo, v. 10, n. 2, p. 276-287, jun. 2007.

SOUZA, N. A.; MARQUES, I. R. Período de estudos e qualidade de vida do estudante de 
enfermagem. Revista Brasileira de Qualidade Vida, Ponta Grossa, v. 2, n. 2, p. 1-8, jul./dez. 2010.

THE WHOQOL GROUP. The World Health Organization Quality of Life Assessment (QOL): position paper from the World Health Organization. Social Science and Medicine, Leicester, v. 41, n. 10, p. 1403-1409, nov. 1995.

TOGNI, A. C.; CARVAlhO, M. J. S. A escola noturna de ensino médio no Brasil. Revista Iberamericana de Educación, Madrid, n. 44, p. 61-78, may./ago. 2007.

VECCHIA, R. D.; RUIZ, T.; BOCCHI, S. M. M. Qualidade de vida na terceira idade: um conceito subjetivo. Revista Brasileira de Epidemiologia, São Paulo, v. 8, n. 3, p. 246-252, set. 2005.

ZANINI, R. R. et al. Prevalência e fatores associados ao consumo de cigarros entre estudantes de escolas estaduais do ensino médio de Santa Maria, Rio Grande do Sul, Brasil, 2002. Cadernos de Saúde Pública, Rio de Janeiro, v. 22, n. 8, p. 1619-1927, ago. 2006. 